\title{
NUEVO DICTAMEN DE AUDITORÍA, UNA RESPUESTA AL NUEVO CONTEXTO O A LIMITACIONES AL MODELO DE CUMPLIMIENTO
}

\author{
NEW AUDIT OPINION, A RESPONSE TO THE NEW \\ CONTEXT OR LIMITATIONS TO THE COMPLIANCE MODEL
}

Recibido: $12 / 10 / 2017$

Aceptado: 15/12/2017

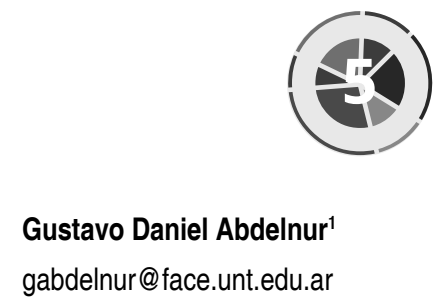

gabdelnur@face.unt.edu.ar

\section{Resumen}

El IAASB aprobó cambios en el informe del Auditor. El resultado fue un mejorado informe que proporciona más transparencia sobre aspectos clave de auditoría y que describe, de mejor forma, lo que una auditoría es y lo que un Auditor hace. El nuevo informe es aplicable para las auditorías de Estados Financieros terminados a partir del 15 de diciembre de 2016 ¿Qué hay de nuevo en el informe del Auditor?

- La sección de "Opinión" se presenta en primer lugar, seguida de la sección "Bases de Opinión", a menos que exista una ley o regulación aplicable que indique lo contrario.

- Declaración sobre la independencia del Auditor y el cumplimiento de las responsabilidades éticas, de acuerdo al código aplicable con referencia al Código de Ética emitido por el IESBA.

- Apartado dedicado a las cuestiones de "Empresa en Marcha", cuando fuera necesario.

- Nueva sección para comunicar los asuntos clave de auditoría (KAM).

Palabras Claves: Opinión - Bases de Opinión - KAM -Negocio en Marcha - NIA

\section{Abstract}

The IAASB approved changes in the Auditor's report. The result was an improved report that provides more transparency on key aspects of audit and describes, in a better way, what an audit is and what an Auditor does. The new report is applicable for audits of financial statements completed as of December 15, 2016. What's new in the Auditor's report?

- The "Opinion" section is presented first, followed by the "Opinion Bases" section, unless there is an applicable law or regulation that indicates otherwise.

- Declaration on the independence of the Auditor and the fulfillment of the ethical responsibilities, according to the applicable code with reference to the Code of Ethics issued by the IESBA (International Ethics Standards Board for Accountants).

- Section dedicated to the issues of Business on the Move, when necessary.

- New section to communicate key audit issues (Key Audit Matters - KAM).

Keywords: Opinion - Opinion Bases - KAM - Business in March - NIA

\footnotetext{
Especialista en Auditoría. Contador Público Nacional. Profesor Adjunto Regular de la Materia "Auditoría". Carrera: Contador Público Nacional Facultad de Ciencias Económicas y Administración - Universidad Nacional de Catamarca, ORCID: 0000-0001-8273-1410
} 


\section{Introducción}

Sin dudas, el informe del Auditor, la principal herramienta de comunicación de este con los usuarios de los Estados Financieros, está experimentando cambios drásticos alrededor del mundo. Un reporte de auditoría "más informativo" es el cambio más visible en la profesión de Auditor externo en más de 50 años.

En la mayoría de los países donde se implementó esta nueva estructura tuvo una reacción positiva de todos los involucrados.

EI IAASB aprobó los cambios en el informe del Auditor en septiembre de 2014 y fue liberado para su adopción a principios de 2015, para los ejercicios económicos finalizados a partir del 15 de diciembre de 2016, inclusive.

El resultado final de este proceso es un nuevo y mejorado informe que proporciona más transparencia sobre aspectos clave de auditoría y que describe, de mejor forma, lo que es una auditoría y lo que hace un Auditor. (IAASB, 2016)

\section{Marco teórico}

\subsection{Definiciones}

Estados financieros con fines generales: son los Estados Financieros preparados de conformidad con un marco de información con fines generales (por ejemplo, las Normas Internacionales de Información Financiera).

Marco de información con fines generales: es un marco de información financiera diseñado para satisfacer las necesidades comunes de información financiera de un amplio espectro de usuarios.

El marco de información financiera puede ser un marco de presentación razonable o un marco de cumplimiento.

El término "marco de presentación razonable" se utiliza para referirse a un marco de información financiera que requiere el cumplimiento de sus requerimientos y, además, reconoce de forma explícita o implícita que, para lograr la presentación razonable de los Estados Financieros, puede ser necesario que la dirección revele información adicional a la requerida específicamente por el marco o reconoce, explícitamente, que puede ser necesario que la dirección no cumpla alguno de los requerimientos del marco para lograr la presentación razonable de los Estados Financieros. Se espera que esto sólo sea necesario en circunstancias extremadamente poco frecuentes.

Un "marco de cumplimiento" se utiliza para referirse a un marco de información financiera que requiere el cumplimiento de sus requerimientos, sin contemplar las posibilidades descritas en los apartados anteriores.

Opinión no modificada (favorable): opinión expresada por el Auditor cuando concluye que los Estados Financieros han sido preparados, en todos los aspectos materiales, de conformidad con el marco de información financiera aplicable.

\subsection{El por qué de la nueva estructura}

Aunque el formato de opinión actual (cumple o no cumple) sigue siendo valioso, no proporciona a los usuarios otros comentarios sobre el proceso de la auditoría. Este nuevo formato está diseñado para ser de gran ayuda para los miembros del Comité de auditoría (en conjunto con el Consejo de Administración y Accionistas) y otros responsables de vigilar la adecuada preparación de los Estados Financieros, al comprender las implicaciones de un documento más informativo, así como la importancia de su rol en el proceso de la auditoría. 
No es de extrañarse que existan muchas preguntas sobre las cuales, los inversionistas y otros usuarios, consideran lo más relevante del nuevo informe de auditoría: Comunicar los asuntos clave de auditoría (IFAC, 2017).

Los KAM proporcionan la oportunidad para compartir las perspectivas del Auditor con los usuarios de los Estados Financieros sobre los asuntos que son de mayor trascendencia sobre la auditoría del período que se informa. Además, y aún más importante, es que estos asuntos fueron probablemente objeto de un diálogo más solido del Auditor con el Comité de auditoría.

Básicamente, lo que se buscó con este cambio fue satisfacer la necesidad de incrementar el valor de la información proporcionada por los Auditores haciendo que la información que proporciona el informe de auditoría sea más relevante para los usuarios (útil para la toma de decisiones).

Los KAM son exigibles para los informes de auditoría de entidades listadas (cotizadas) o en aquellos casos que así lo establezcan disposiciones legales o por voluntad del Auditor.

\subsection{Nueva estructura del reporte}

El informe de auditoría de Estados financieros con fines generales tiene como objetivo emitir una opinión sobre el cumplimiento razonable o no que estos hacen del marco de información financiera aplicable. La estructura de este es la siguiente:

Tabla 1. Estructura del reporte

\begin{tabular}{|l|l|}
\hline Título & Responsabilidad del Auditor en relación con la auditoría \\
Destinatario & de los Estados Financieros \\
Opinión & Otras responsabilidades de información \\
Fundamento de la opinión & Nombre del socio del encargo \\
Empresa en funcionamiento & Firma del Auditor \\
Cuestiones clave de la auditoría & Dirección del Auditor \\
Responsabilidades en relación con los Estados & Fecha del informe de auditoría \\
Financieros. & \\
\hline
\end{tabular}

Elaboración: el autor.

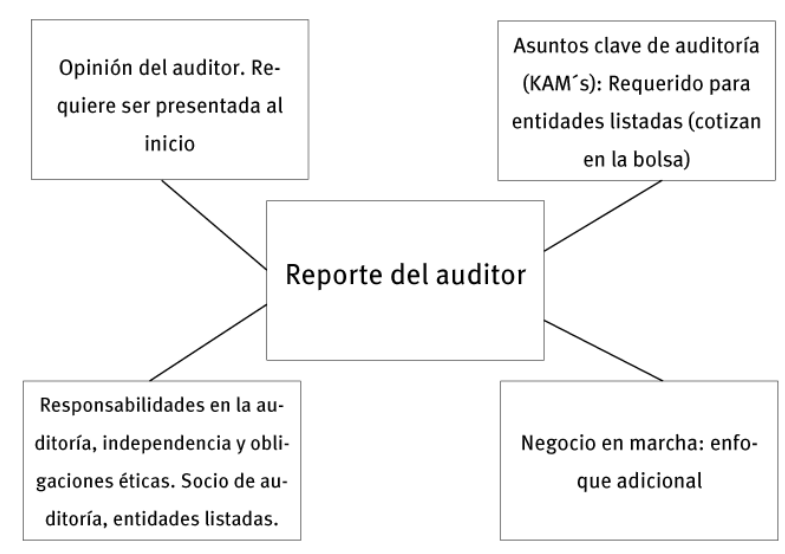

Figura 1. Nueva estructura del reporte del Auditor 


\subsection{Marco normativo del reporte del Auditor}

De igual forma, durante el 2015 fueron aprobadas modificaciones a las siguientes ISA (descritas en la tabla

2) para conformarlas con los cambios efectuados a la ISA 700.

Tabla 2. Formación de la opinión y dictamen sobre los Estados Financieros

\begin{tabular}{|c|c|c|c|}
\hline $\begin{array}{l}\text { ISA } 701 \text { (nueva) } \\
\text { Nueva sección: } \\
\text { Comunicar asuntos } \\
\text { clave de Auditoria. }\end{array}$ & $\begin{array}{c}\text { ISA } 705 \text { (modificada) } \\
\text { Modificaciones a la } \\
\text { opinión del Auditor. }\end{array}$ & $\begin{array}{l}\text { ISA } 570 \text { (modificada) } \\
\text { Mejora en el informe } \\
\text { del Auditor relativo a } \\
\text { negocio en marcha. }\end{array}$ & $\begin{array}{c}\text { ISA } 720 \text { (modificada) } \\
\text { Nueva sección: } \\
\text { Responsabilidad del } \\
\text { Auditor en relación con } \\
\text { otro tipo de información } \\
\text { que se incluye en el } \\
\text { informe anual de la } \\
\text { entidad. }\end{array}$ \\
\hline
\end{tabular}

Elaboración: El autor

\section{Discusión y resultados}

\section{1 ¿Qué hay de nuevo en el informe del Auditor?}

a. La sección de "Opinión" se presenta en primer lugar, seguida de la sección "Bases de Opinión", a menos que exista una ley o regulación aplicable que indique lo contrario.

b. Declaración sobre la "Independencia del Auditor" y el cumplimiento de las "Responsabilidades éticas", de acuerdo con el Código aplicable con referencia al "Código de ética". (IESBA, 2018).

c. Nueva sección para comunicar los "Asuntos clave de auditoría" (KAM). Los asuntos claves de auditoría son aquellas cuestiones que, a juicio del Auditor, fueron de gran importancia en la auditoría de los Estados Financieros del ejercicio en curso. Esta sección de KAM solo será aplicable para informes de auditoría de entidades públicas y otras entidades de interés público.

d. La mejora en el informe relativo a "Negocio en marcha", incluye:

- Descripción de las respectivas responsabilidades de la gerencia y el Auditor sobre

- negocio en marcha.

- Una sección por separado cuando existe incertidumbre material y es adecuado

- revelarlo bajo el título "incertidumbre material relacionada con Negocio en Marcha".

- Nuevo requerimiento para la adecuada revelación de si la entidad está

- significativamente cerca de tener en duda su capacidad de continuar como "Negocio en marcha", desde el punto de vista del marco financiero aplicable y cuando existen eventos o condiciones identificados.

- La descripción de las responsabilidades propias del Auditor y las características

- fundamentales de la auditoría. Ciertos componentes de la descripción de las responsabilidades del 
Auditor pueden presentarse en un apéndice al informe del Auditor.

¿Desde cuándo son efectivos estos cambios? El nuevo informe del Auditor y las normas aplicables son efectivas para las auditorías de los Estados Financieros correspondientes a ejercicios terminados a partir del 15 de diciembre de 2016, inclusive.

\subsection{Cuestiones clave de la auditoría KAM - ISA 701}

Son cuestiones que, a juicio profesional, fueron las de mayor importancia en la auditoría de los Estados Financieros del período actual. Las cuestiones clave de auditoría son seleccionadas de cuestiones comunicadas a los encargados del gobierno de la entidad.

El Auditor debe seleccionar, de las cuestiones comunicadas a los encargados del gobierno, aquellas que requirieron de su atención significativa al llevar a cabo la auditoría, teniendo en cuenta lo siguiente:

- Las áreas de mayor riesgo valorado de incorrección material o de riesgos

- significativos identificados de conformidad con la ISA 315.

- Los juicios significativos del Auditor relativos a áreas en los Estados Financieros,

- que involucran juicios significativos de la dirección, incluyendo estimaciones contables que se han identificado como que tienen una alta incertidumbre en la estimación.

- El efecto en la auditoría de eventos o transacciones significativas ocurridas durante

- el período.

- El Auditor debe determinar cuáles de las cuestiones determinadas fueron de mayor

- importancia en la auditoría de los Estados Financieros del período actual y por lo tanto son cuestiones clave de la auditoría.

- En algunas circunstancias limitadas, es posible que no existan KAM a comunicar. Se

- requiere que el Auditor incluya cada KAM a menos que:

- Exista una ley o reglamento que impida su divulgación pública.

- En circunstancias excepcionales, cuando el Auditor determine que un asunto no

- debe ser comunicado, pues las consecuencias adversas de hacerlo podrían superar razonablemente los beneficios del interés público de dicha publicación.

- Ejemplo de KAM son los siguientes:

- Plusvalía que debe ser probada por deterioro y su prueba reviste complejidad.

- Instrumentos financieros para cuya medición se usaron modelos internos ya que no

- tienen una cotización pública.

- Valoración de activos y pasivos en un plan de pensiones de beneficios definidos.

- Reconocimiento de ingresos de un producto que tiene una garantía cuyo plazo hace

- dificultosa las provisiones.

- Provisiones por restructuración.

\subsection{Negocio en marcha - ISA 570}

El Auditor tiene la responsabilidad de obtener evidencia de auditoría suficiente y adecuada, y concluir sobre la idoneidad de la utilización por parte de la dirección de las bases contables de una empresa en funcionamiento para la preparación de los Estados Financieros, así como, determinar si, con base en la evidencia obtenida, existe alguna incertidumbre de la entidad para continuar como empresa en funcionamiento.

Estas responsabilidades existen aún en el caso que el marco de información financiera utilizado para la preparación de los Estados Financieros no contenga como requerimiento explícito que la dirección realice una valoración específica de la capacidad de la entidad para continuar como empresa en funcionamiento.

El Auditor deberá informar respecto al resultado de su conclusión. 
Tabla 3. Ejemplo de revelación en el informe del Auditor

\section{Responsabilidad del Auditor:}

Concluir sobre lo apropiado del uso de la dirección de las bases contables de empresa en funcionamiento $y$, con base en la evidencia obtenida, si existe una incertidumbre material relativa a eventos o condiciones que puedan originar una duda significativa sobre la capacidad de la Compañía para continuar como empresa en funcionamiento. Si concluimos que existe una incertidumbre material, se nos pide llamar la atención en nuestro informe del Auditor sobre la información a revelar respectiva a los Estados Financieros o, si dicha información a revelar es insuficiente para modificar nuestra opinión. Nuestras conclusiones se basan en la evidencia de auditoría obtenida hasta la fecha de nuestro informe del Auditor. Sin embargo, hechos o condiciones futuros pueden causar que la Compañía deje de ser una empresa en funcionamiento.

\section{Responsabilidad del Auditor:}

Los cambios en las ISA 700 y 701 incluyen una descripción más amplia de las responsabilidades de la administración y del Auditor, incluidas aquellas relativas a "Negocio en marcha".

Debido a esto, dichas secciones pueden presentarse por separado en un informe adicional.

\section{Elaboración: El autor}

\subsection{El estado actual del cambio}

La comunicación de los KAM no es un reemplazo o complemento a la perspectiva de la administración plasmada en sus Estados Financieros, ni se busca llenar los huecos en revelaciones que se perciban como incompletas u omitidas en los Estados Financieros (no son sustitutos de una opinión modificada). La consideración del Auditor sobre lo apropiado y adecuado de las revelaciones es parte del trabajo para emitir una opinión sobre los Estados Financieros.

Es claro que frecuentemente habrá una relación estrecha entre los KAM y las áreas de complejidad o de juicios significativos en los Estados Financieros, debido a la naturaleza misma de la auditoría, la cual está basada en un enfoque que dirige la atención del Auditor a los asuntos en los que existe un mayor riesgo de incorrección material.

La intención de los KAM es proveer transparencia a los usuarios sobre cómo estos asuntos afectaron el plan de auditoría y cómo se trataron dichos asuntos en los Estados Financieros.

Respecto a los efectos en la relación y diálogo entre el Auditor y el Comité de auditoría/Consejo de Administración, la inclusión de los KAM enriquece la comunicación entre ambas partes del encargo e incrementa la atención por parte de la Compañía sobre las revelaciones referidas en el informe del Auditor.

La experiencia viene señalando que no hay diferencias de opinión acerca de los KAM a incluirse en el informe de auditoría, siempre y cuando la Administración, el Comité de auditoría y el Auditor entiendan el objetivo de proveer mayor transparencia sobre el proceso del encargo y que haya existido un diálogo continuo durante el proceso de auditoría, desde la etapa de planeación sobre aquellos asuntos candidatos a convertirse en KAM.

De mucha utilidad será para la Compañía, observar un borrador del informe de auditoría tan pronto como sea posible y antes de su formalización, no implicando esta práctica la idea de "consensuar" el mismo, en el mal sentido de la palabra.

Las Normas de auditoría requieren que el Auditor comunique a los encargados del gobierno corporativo ciertos asuntos, incluyendo el punto de vista sobre aspectos cualitativos de las prácticas contables, estimaciones y revelaciones. El objetivo de esta comunicación es proveer a aquellos responsables de 
supervisar la información financiera, cualquier observación incluyendo preocupaciones que el Auditor tenga sobre estos temas.

Algunos usuarios han recibido de manera positiva esta transparencia adicional de los KAM y los comentarios del Auditor. Otros están preocupados por una posible interpretación errónea como opiniones individuales de los temas y que no se revise la opinión sobre los Estados Financieros en su conjunto.

Este reporte más informativo puede apoyar la función de gestión de la administración de la Compañía, mediante la creación de un vínculo más estrecho entre la función de auditoría, las responsabilidades de la alta dirección y el Comité de auditoría.

\subsection{El impacto del nuevo reporte en el Auditor y su tarea}

El número de KAM en el informe de auditoría es el resultado de una evaluación que implica el juicio del Auditor y la importancia relativa de los mismos. Las normas de auditoría no prescriben o limitan el número de KAM a ser reportados.

Como mencionamos anteriormente en esta ponencia, la intención del Auditor al comunicar los KAM es proporcionar mayor transparencia a los usuarios, por ende, estos asuntos deben ser incluidos en el reporte del Auditor, a menos que una ley o regulación lo impida. De ninguna manera, un KAM podrá ser un elemento que supla alguna deficiencia cometida por la empresa en la preparación de los Estados Financieros; estas situaciones derivarán en una modificación de la Opinión de auditoría.

En circunstancias extremadamente raras, el Auditor puede determinar que el asunto no debe ser comunicado debido a las consecuencias adversas que puede provocar hacerlo, en comparación con los beneficios de hacer en el interés del público.

La principal preocupación actualmente es evitar que los KAM comiencen a suplir la emisión de una opinión modificada (con salvedades, adversa o abstención de emitirla), en lugar de cumplir con su objetivo primordial que es proporcionar información útil y pertinente para los inversionistas.

Sobre la calidad del encargo de auditoría, este reporte más informativo brinda más transparencia acerca de qué es una auditoría y cómo se lleva a cabo. Esto representa al menos un efecto positivo sobre la calidad de la auditoría. Probablemente, el Auditor no ejecutará procedimientos adicionales o diferentes, pero se preguntará si lo que informa a los usuarios sobre cómo se atacaron los aspectos más importantes está adecuadamente plasmado en sus papeles de trabajo.

Respecto al tiempo y la extensión del encargo, el nuevo reporte de auditoría representa un impacto, pero si el Auditor sabe manejarlo a lo largo del proceso de la auditoría, este no será significativo ni impactará en sus honorarios.

El principal problema que se observa en algunos países donde coexisten las normas internaciones con normas locales, es la diferencia en los reportes de auditoría que presentan las empresas que cotizan en bolsa respecto de las que no. Esto ocasiona un problema de comparabilidad y uniformidad y hasta la creencia que un encargo podría ser de mayor calidad que el otro.

El IAASB sigue de cerca las reacciones, considerando si otras mejoras pueden dar mayor valor al informe del Auditor, siempre buscando que sean útiles para el público inversionista.

\subsection{Ejemplo de informe de auditoría con la nueva estructura}

Informe de los Auditores independientes al Consejo de Administración y Accionistas de la Entidad XX SA

Opinión

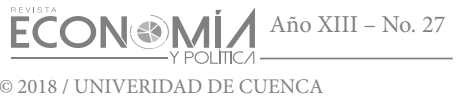


Hemos auditado los Estados Financieros adjuntos de la Entidad XX SA (la Entidad), los cuáles comprenden los estados de situación financiera al 31 de diciembre de 2017 y 2016, y los estados del resultado, los cambios en el capital contable y de flujos de efectivo, correspondientes a los ejercicios que terminaron en dichas fechas, así como un resumen de las políticas contables significativas y otra información explicativa. En nuestra opinión, los Estados Financieros adjuntos presentan razonablemente, en todos los aspectos significativos, la situación financiera de la Entidad XX SA al 31 de diciembre de 2017 y 2016, así como sus resultados y sus flujos de efectivo correspondientes por los ejercicios que terminaron en dichas fechas, de conformidad con las Normas Internacionales de Información Financiera.

\section{Bases de la Opinión}

Nuestra auditoría fue efectuada de acuerdo con las Normas Internacionales de auditoría. Nuestras responsabilidades bajo estas normas se describen con más detalle en la sección de responsabilidades del Auditor para la auditoría de los Estados Financieros de este informe. Somos independientes de la Entidad XX SA de acuerdo con las disposiciones del Código de Ética para Contadores Públicos emitido por el Consejo de Normas Internacionales de Ética (IESBA), y hemos cumplido con nuestras otras responsabilidades éticas en conformidad con estos requisitos. Consideramos que la evidencia de auditoría que hemos obtenido es suficiente para proporcionar una base razonable para nuestra opinión.

\section{Asuntos Clave de auditoría}

Asuntos Clave de auditoría son aquellos asuntos que fueron de mayor importancia en nuestra auditoría de los Estados Financieros del período actual. Estas cuestiones fueron abordadas en el marco de nuestra auditoría considerados como un todo, y en la formación de nuestra opinión al respecto, por lo que no representan una opinión por separado sobre estos asuntos. La cantidad de ingresos y ganancias reconocidos en el ejercicio por la venta del producto "Z" y los servicios post venta, dependen de la adecuada valoración de si a cada contrato post venta a largo plazo se lo vincula o no del contrato de la venta del producto. Como los acuerdos comerciales pueden ser complejos, se aplica el juicio significativo en la selección de la base contable en cada caso. En nuestra opinión, el reconocimiento de ingresos es un riesgo significativo para nuestra auditoría ya que la Entidad podría reconocer inapropiadamente las ventas del producto " $Z$ " y los acuerdos de servicio a largo plazo como un único acuerdo para efectos contables. Esto puede conducir a que los ingresos y las ganancias sean reconocidos de manera anticipada ya que el margen en el acuerdo de servicio a largo plazo suele ser mayor que el margen en el contrato del producto "Z" dentro del contrato de venta.

Nuestros procedimientos de auditoría para cubrir el riesgo significativo en relación con el reconocimiento de ingresos incluyeron:

1. Pruebas de controles, con la asistencia de nuestros especialistas en Tecnologías de la Información, incluyendo entre otros, aquellos sobre la fuente de información de datos de las campañas publicitarias individuales, sus términos y precios; la comparación de dichos términos y precios contra los contratos relativos a las agencias de publicidad existentes; y su relación con los datos observados; $y$

2. Análisis detallados de los ingresos y el momento de su reconocimiento, en base a las expectativas derivadas de nuestro conocimiento de la industria y de los datos de mercado externos, dando seguimiento a las variaciones de nuestras expectativas.

En la Nota 3, a los Estados Financieros se incluyen las revelaciones de la Entidad sobre el reconocimiento de ingresos, lo que explica específicamente el tratamiento de los contratos de servicios post venta utilizados en los supuestos clave para evitar un reconocimiento de ingresos anticipado.

Otra información incluida en el documento que contienen los Estados Financieros auditados 
La administración es responsable de la otra información que acompaña a los Estados Financieros objeto de nuestro encargo de auditoría. La otra información comprende al reporte anual que se presenta según las disposiciones de carácter general aplicables a los emisores de valores en Argentina.

Nuestra opinión sobre los Estados Financieros no cubre la otra información y no podemos expresar cualquier tipo de opinión al respecto.

En relación con nuestra auditoría de los Estados Financieros, es nuestra responsabilidad leer la otra información y, al hacerlo, considerar si esta es materialmente consistente con los Estados Financieros o con nuestros conocimientos obtenidos en la auditoría, o de lo contrario si parece estar materialmente distorsionada. Si, sobre la base del trabajo que hemos realizado, podemos concluir que existe una inexactitud importante de esta otra información, estamos obligados a notificar este hecho. No tenemos nada que informar a este respecto.

\section{$\underline{\text { Responsabilidades del Auditor para la auditoría de los Estados Financieros (ver Anexo A) }}$}

Nuestros objetivos son obtener una seguridad razonable acerca de si los Estados Financieros como un todo están libres de incorrecciones materiales, ya sea por fraude o error, y emitir nuestra opinión. Seguridad razonable es un alto nivel de seguridad, pero no es garantía que una auditoría realizada de acuerdo con Normas Internacionales de auditoría siempre va a detectar incorrecciones materiales, cuando estas existan. Incorrecciones pueden surgir por fraude o error y se considera material si, individualmente o en conjunto, puede esperarse razonablemente que influyan en las decisiones económicas de los usuarios, tomadas sobre la base de los Estados Financieros.

Responsabilidades de la administración y los encargados del gobierno en relación con los Estados Financieros

La administración de la Entidad es responsable de la preparación y presentación razonable de los Estados Financieros adjuntos, de conformidad con las Normas Internacionales de Información Financiera, así como del control interno que la administración de la Entidad considere necesario para permitir la preparación de los Estados Financieros libres de incorrecciones materiales debidas a fraude o error.

En la preparación de los Estados Financieros, la administración es responsable de evaluar la capacidad de la Entidad para continuar como un negocio en marcha, revelando, según corresponda, los asuntos relacionados, salvo que la administración se proponga liquidar la Entidad o cesar sus operaciones, o no tenga otra alternativa más realista que hacerlo.

Los encargados de gobierno son responsables de supervisar el proceso de presentación de los informes financieros de la Entidad.

XX y Asociados

(Firma del socio)

CP Cosme Fulanito

de

de 2018 (CCEA, 2017) 


\section{Anexo A}

Como parte de una auditoría de acuerdo con las Normas Internacionales de auditoría, aplicamos el juicio profesional y mantenemos el escepticismo profesional durante toda la auditoría. Asimismo:

- Identificamos y evaluamos los riesgos de errores significativos en los Estados Financieros, ya sea por fraude o error, diseñamos y realizamos procedimientos de auditoría que responden a esos riesgos, obtenemos evidencia de auditoría suficiente y apropiada para proporcionar una base para nuestra opinión. El riesgo de no detectar una incorrección material resultante de fraude es mayor que de errores, pues el fraude puede implicar la colusión, falsificación, omisiones intencionales, falseamiento o la rescisión del control interno.

- Obtenemos un entendimiento del control interno relevante para la auditoría con el fin de diseñar procedimientos de auditoría que sean apropiados en las circunstancias, pero no con el propósito de expresar una opinión sobre la efectividad del control interno de la entidad.

- Evaluamos lo adecuado de las políticas contables utilizadas y la razonabilidad de las estimaciones contables y revelaciones relacionadas efectuadas por la administración.

- Concluimos sobre el uso adecuado por la administración del supuesto de negocio en marcha y en base a la evidencia de auditoría obtenida, si existe o no relaciones con eventos que puedan proyectar una duda importante sobre la capacidad de la entidad para continuar como un negocio en marcha. Si llegamos a la conclusión que existe una incertidumbre material, estamos obligados a llamar la atención en nuestro informe de auditoría de las revelaciones relacionadas en los Estados Financieros o, si tales revelaciones son insuficientes, modificar nuestra opinión. Nuestras conclusiones se basan en la evidencia de auditoría obtenida hasta la fecha de nuestro informe de auditoría. Sin embargo, eventos futuros o condiciones pueden causar que la entidad no pueda continuar como un negocio en marcha.

- Evaluamos la presentación, estructura y contenido de los Estados Financieros, incluyendo las revelaciones, y si los Estados Financieros representan las operaciones subyacentes y eventos en una forma que logre presentación razonable, obteniendo suficiente evidencia apropiada de auditoría respecto de la información financiera de las actividades comerciales de la Entidad como expresar una opinión sobre los Estados Financieros. Somos responsables de la dirección, supervisión y ejecución de la auditoría de la Entidad. Somos los únicos responsables de nuestra opinión de auditoría.

- Nos comunicamos con los encargados del gobierno en relación con, entre otras cosas, al alcance y el momento de la auditoría y los resultados de auditoría importantes, incluidas posibles deficiencias significativas en el control interno que identificamos durante nuestra auditoría.

- También proporcionamos los encargados del gobierno con una declaración que hemos cumplido con los requisitos éticos relevantes con respecto a la independencia y hemos comunicado todas las revelaciones y otros asuntos que puedan soportarla.

- A partir de las comunicaciones con los encargados del gobierno, determinamos aquellos asuntos que eran de la mayor importancia en la auditoría de los Estados Financieros del período actual y por lo tanto son los Asuntos Clave de auditoría.

- Describimos estos asuntos en nuestro informe de auditoría a menos que la ley o el reglamento se opongan a la divulgación pública de los mismos o cuando, en circunstancias extremadamente raras, determinamos que un asunto no debe ser comunicado en nuestro informe debido a posibles consecuencias adversas.

\section{Conclusiones y recomendaciones}

- Estas modificaciones jerarquizan el informe de auditoría y la tarea del Auditor. Desde la experiencia, 
se puede afirmar que la inclusión de los KAM no da lugar a ningún tipo de hecho que contraríe las normas y las buenas prácticas profesionales. Por el contrario, incrementó el interés acerca del trabajo del Auditor por parte de los directivos y los usuarios

- Lo negativo es que las KAM son exigibles solo para las auditorías de empresas que cotizan en bolsa. Esto produce y genera la idea que la auditoría en un caso podría ser de mayor calidad que en el otro. Esto se acentúa en aquellos países en los que hay un doble marco normativo: es decir, normas para entes que cotizan en bolsa (NIIF) y normas para aquellos que no lo hacen (Normas locales). Como recomendación, es necesario generalizar la aplicación de esta estructura para todo tipo de empresas, pues lo intereses de los propietarios y demás interesados en la información de los Estados Financieros son semejantes.

- En Argentina coexisten normas internacionales y nacionales. Las internacionales se aplican para las empresas que cotizan en bolsa y las nacionales para las no cotizables (con independencia de su tamaño). Los informes de auditoría conforme las normas nacionales se estructuran y redactan conforme el modelo tradicional de informe, antes de las modificaciones, y para esas empresas no existe la posibilidad de incluir los KAM en los reportes del Auditor. Recomendamos modificar las normas contables profesionales locales (Resolución Técnica № 37 de la Federación Argentina de Consejos Profesionales en Ciencias Económicas), de aplicación para auditorías en empresas que no cotizan en bolsa, de manera tal que los informes incluyan los KAM.

- Existe una necesidad de generalizar la aplicación de estas normas para todo tipo de entes, como una forma de jerarquizar la profesión de la auditoría. Esta tarea debería hacerse desde la etapa educativa, es decir, trabajar con los alumnos de las carreras de grado de Contador Público y afines, de manera que la semántica para expresar los KAM cuente con el correcto adiestramiento y se encuentre incorporada desde temprana edad.

- Para terminar, una reflexión y un consejo. La reflexión: el cambio en el informe de auditoría va a suponer para los Auditores un nuevo reto técnico (sin duda superable por la profesión Auditora como ha ocurrido en tantas otras veces que se ha producido una modificación legal). Adicionalmente, para las entidades auditadas, llega el momento de aceptar y consentir que la nueva información (más explicativa y extensa) quede plasmada en la redacción del informe elaborado, que en numerosos casos desembocará en fuente de conflictos y que requerirá la maestría habitual del buen Auditor para mantener el difícil equilibrio entre independencia profesional, legalidad y conformidad del cliente. El consejo: siendo importante la lectura del contenido íntegro del informe de auditoría, es especialmente interesante que le dediquen atención a la opinión y a las CCA o AMRA que consten en el mismo. 


\section{Referencias bibliográficas}

- CCEA. (05 de 06 de 2017). Colegio de Contadores, Economistas y Administradores de Uruguay. Obtenido de http://www.ccea.org.uy/docs/NIAS/Anexo\%202.pdf

- IAASB. (2016). NIA ${ }^{\mathrm{TM}} 800$ (Revisada), Consideraciones especiales-Auditorías de estados financieros preparados de acuerdo con los marcos de propósito general. Nueva York: IFAC GLOBAL.

- IESBA. (2018). International Code of Ethics for Professional Accountants. New York: IFAC.

- IFAC. (13 de diciembre de 2017). IFAC.ORG. Obtenido deAuditorReporting Standards Implementation: Key Audit Matters: https://www.ifac.org/global-knowledge-gateway/audit-assurance/discussion/ auditor-reporting-standards-implementation-key 